\section{Occupational infection risks in interventional radiology}

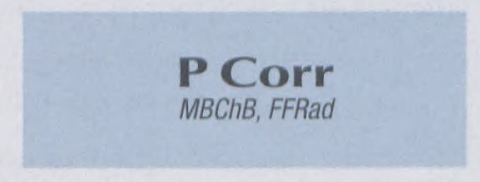

ccupational exposure to viral material during invasive procedures is a major concern to health professionals in many countries. ${ }^{1}$ South Africa has a particularly high prevalence of HIV and hepatitis B and C infection in the population..$^{2,3}$ Radiologists, radiographers and nurses who perform and assist with angiography or interventional procedures are at risk through needle stick injuries or corneal splashes from blood. ${ }^{4,5}$ It is important that radiologists be aware of the risk, how to reduce the risk and what to do after needle stick injuries. ${ }^{6}$

\section{Risk of HIV infection}

South Africa currently has an epidemic of HIV infection from heterosexual spread. The latent period from seroconversion to the development of the acquired immunodeficiency syndrome (AIDS) is approximately ten years. Many of our patients requiring interventional or invasive procedures will be incidentally seropositive. The HIV positivity rate varies from less than $5 \%$ in the Western Cape to 23\% in routine antenatal clinic screening in KwaZulu Natal. ${ }^{2}$ Patients with clinical AIDS are more likely than other patients to have invasive procedures such as biopsies, drainage of intra-abdominal collections or abscesses and angiography for arterial occlusions and aneurysms.

It is very important for the radiologist, radiographer and nurse to be aware of the occupational risk from HIV. Data about the risk of seroconversion are derived mostly from studies in the United States reported by the Center for Disease Control and Prevention (CDC). Occupational infection has been definitely 


\section{from page 17}

documented in 52 health professionals and probably in 111 other workers. ${ }^{7}$ The majority of the documented cases $(90 \%)$ involved blood exposure. Eighty-seven percent of all these cases followed needle stick injuries. The CDC reviewed 25 studies of HIV seroconversion from 1983 to 1994 recently. ${ }^{6,7}$ The documented risk for percutaneous exposure is $0.3 \%$ ( 21 infections in 6498 documented exposures).

A retrospective review comparing incidents involving seroconversion versus those workers who did not seroconvert, found that those workers who seroconverted had the following increased risks: a deep percutaneous injury, visible blood on the instrument or needle, the instrument was used for arterial or venous access, and the patient was terminally ill. Seroconversion is more likely with a large volume of blood and a high viral load. ${ }^{8}$ The risk for mucous membrane exposure was estimated to be $0.1 \%$ and for intact skin exposure less than $0.1 \%{ }^{8}$

\section{Risk of hepatitis $B$ infection}

Hepatitis B is the most common occupational infection world-wide. ${ }^{1}$ In South Africa the prevalence of hepatitis envelope antigen ( $\mathrm{HBeAg}$ ) is particularly high, in the region of $10 \% .{ }^{3}$ Hepatitis B is extremely infectious because of much higher circulating blood viral antigen levels ( $10^{13}$ viral particles per $\mathrm{ml}$ ) than with HIV. The virus survives in dried blood for up to a week, unlike HIV, which dies within minutes. The risk of seroconversion is $12 \%$ in non-immunised workers after percutaneous inoculation of the hepatitis surface antigen $(\mathrm{HBsAg})$. The risk increases to
$30 \%$ after exposure to the envelope antigen of the virus ( $\mathrm{HBeAg}) .{ }^{9} \mathrm{How}-$ ever the risk of seroconversion is minimal in the fully immunised worker. ${ }^{10}$

It is essential that all health workers have full immunisation for hepatitis $B$. The recombinant $D N A$ vaccine is proven to be extremely safe and effective. It is critically important that a protective antibody level greater than $10 \mathrm{mIU} / \mathrm{ml}$ be documented in all workers to ensure effective immunisation. Immunisation failure is due to a variety of reasons, usually including incorrect administration of the vaccine or failure to receive the 6 month and 5 year boosters. Health workers should not be allowed to work in the interventional room without immunisation.

Infection with hepatitis B may result in chronic hepatitis in $10-25 \%$ of patients. The risk of resultant cirrhosis and hepatoma is about $10 \%$. The CDC estimates that in the United States 8700 health professionals are infected every year with hepatitis B. This results in up to 190 deaths from hepatitis per year. ${ }^{10}$

\section{Risk of hepatitis $C$ infection}

Hepatitis $C$ is the most common cause of non- $\mathrm{A}$ and non-B hepatitis infections from blood transfusions. The prevalence of hepatitis $C$ is in the region of $1.2 \%$ in donated blood in South Africa. ${ }^{3}$ In the USA the prevalence is $0.6 \%$. The risk of developing chronic liver disease is much higher than with hepatitis B, with $85 \%$ of patients with acute infections remaining chronically infected. Twenty percent of these patients develop chronic liver disease and cirrhosis. The estimated risk of infection following a percutaneous injury is between 6 and
$10 \%$, making it more infectious than HIV but far less infectious than hepatitis B. No vaccine has been developed yet and there is no obvious benefit in giving hyperimmune globulin for post exposure prophylaxis.

\section{Precautions}

Most precautions involve common sense. Remember to treat every patient as infectious until proven otherwise. Normal precautions include:

1. Do not recap needles - this is the single most important precaution you can take. Most needle sticks occur during recapping of the needle. Great care must be used when reinserting the stylet of the Potts needle into the cannula. Never hand a sharp needle or instrument to an assistant. Always place sharps on the tray first.

2. Do not use glass syringes for contrast injections because of the risk of shattering. Polycarbonate syringes are much stronger than conventional plastic syringes.

3. Ensure adequate lighting over the working area during the procedure. There has been shown to be a risk in many interventional rooms where lighting is poor.

4. Always wear latex gloves, preferably double-gloving. Occult perforations occur in $10 \%$ of gloves during interventional procedures, increasing to $23 \%$ in those procedures lasting more than two hours. Double gloving reduces this risk substantially, particularly during long procedures.

5. Always wear a sterile gown, mask and transparent face shield when performing procedures, particularly angiography. It is extremely important to remember to use facemasks with 


\section{from page 18}

the attached plastic shield to cover the eyes to prevent corneal splashes.

6. The modified arteriography Pott's needle with a vacuum side arm prevents blood splashes during arterial puncture. It is now available in South Africa and costs R15 more than the conventional needle.

7. A closed angiographic flush system avoids blood splashes when flushing syringes. One can aspirate heparinised saline and contrast and dispose of bloody fluid into a closed drainage container. Try to avoid using open bowls on the tray, as there is a substantial risk of splashes. The closed flush system is available commercially. However one could probably make one's own from drip tubing, a three way tap, a vacolitre of normal saline and a urine bag for blood disposal.

8. Remember to cover the image intensifier with sterile plastic to prevent blood splashes.

\section{Exposure to blood}

If you are splashed with blood, wash the exposed skin with soap and water, and flush mucous membranes and eyes with water. If a sharp injury occurs, you must notify the infection control officer in your hospital. Take blood from the patient with his or her written consent to check the HIV status. Your blood will be required for baseline HIV testing. If the patient is HIV positive, postexposure prophylaxis should be started as soon as possible, preferably within 1 to 2 hours. Early prophylaxis is critical in preventing seroconversion. The window of opportunity to prevent viral spread is a few hours. Zidovudine (AZT) $200 \mathrm{mg}$ tds and Lamivudine (Epivir) $150 \mathrm{mg}$ bd for four weeks is currently recommended by the $\mathrm{CDC}$ in the
USA (CDC website address for current information is http:// www.cdc.gov ). ${ }^{11}$ It is important that early counselling of the risks of seroconversion commences as soon as possible. Testing for HIV should be repeated at 6 weeks, 3 months and 6 months. This prophylaxis is highly effective, reducing the risk of seroconversion by $79 \%$. If the patient is positive for hepatitis $B$, and you are not fully immunised, immune globulin must be administered within 24 hours and the hepatitis B booster given. There is no evidence that hyperimmune globulin prevents hepatitis $\mathrm{C}$ infection.

\section{Conclusions}

It is critically important that all health care workers, especially radiologists performing interventional procedures, be aware of the risk of infection in their daily practice. Precautions involve common sense and care when handling sharps. You must be aware of your local hospital policy on post exposure prophylaxis, whom to contact and try to educate your working colleagues on safe working conditions in the interventional room.

\section{Acknowledgements}

I would like to thank Professor Susan Wall, Interventional Radiology, University of California, San Francisco, USA for permission to use her data in preparation for this article.

\section{References}

1. Gerberding JL. Management of occupational exposures to blood borne viruses. NEJM 1995; 332: 444-51.

2. Williams B, Campbell C. Understanding the epidemic of HIV in South Africa: analysis of the antenatal clinic screening data. S Afr Med J 1998; 88(3): 249-51.
3. Kew MC. Progress towards the comprehensive control of hepatitis B in Africa: a view from South Africa. Gut 1996; 38(supp 2: S31-36).

4. McWilliams RG, Blanshard KS. The risk of blood splash contamination during angiography. Clinical Radiology 1994; 49: 59-60.

5. Hansen ME, Miller GL, Redman HC, McIntire DD. Needle stick injuries and blood contacts during invasive radiologic procedures: frequency and risk factors. AJR 1993; 160: 1119-22.

6. Wall SD, Howe JM, Sawhney R. Human immunodeficiency virus infection and hepatitis: biosafety in radiology. Radiology 1997; 205(3): 619-28.

7. Centers for Disease Control and Prevention. Case control study of HIV seroconversion in health care workers after percutaneous exposure to HIVinfected blood-France, UK, USA January 1988-August 1994. MMWR $1995 ;$ 44: 929-933.

8. Centers for Disease Control and Prevention- HIV/AIDS surveillance report: US HIV and AIDS cases reported through December 1996-97; $8(2): 20-21$.

9. Zuckerman AJ. Occupational exposure to hepatitis B virus and human immunodeficiency virus. A comparative risk analysis. Am J Infect Control 1995; 23: 28 .

10. Center for Disease Control and Prevention. Recommendations for prevention of transmission of immunodeficiency virus and hepatitis $B$ virus to patients during exposure prone invasive procedures. MMWR $1991 ; 40: 1-9$.

11. Center for Disease Control and Prevention. Public health service guidelines for the management of health care worker exposure to HIV and recommendations for post exposure prophylaxis. MMWR 1998; 47: $1-10$ 